\title{
Mind-Modulated Music in the Mind Attention Interface
}

\author{
Ben Swift, James Sheridan, Yang Zhen and Henry J. Gardner \\ Department of Computer Science, FEIT \\ ANU College of Engineering and Computer Science \\ Australian National University \\ Canberra, ACT 0200, Australia \\ benjamin.swift@yahoo.com.au,James.Sheridan@anu.edu.au, \\ u4241497@anu.edu.au, Henry.Gardner@anu.edu.au
}

\begin{abstract}
A recent study of electroencephalogram (EEG) activity associated with musical cognition has suggested a correlate for the amount of active musical processing taking place in the brains of musicians. Using a version of this measure, we have built a new brain computer interface which harnesses the "natural" brain activity of musicians to mold and modulate music as it is being composed and played. This computer music instrument is part of a system, the Mind Attention Interface, which provides an interface to a virtual reality theatre using measures of a participant's EEG, eye-gaze and head position. The theatre itself, and its spatialised sound system, closes a feedback loop through the mind of the participant.
\end{abstract}

\section{Categories and Subject Descriptors}

I.3.7 [Three-Dimensional Graphics and Realism]: Virtual Reality; J.5 [Arts and Humanities]: Performing arts

\section{Keywords}

computer music, brain computer interface, EEG, functional connectivity

\section{INTRODUCTION}

Brain-Computer Interfaces (BCIs) offer the ability to interface with computer systems through direct measurements of neural activity. There are many different approaches to this problem, and several helpful reviews are available (for example [15]).

At the heart of any BCI design is the determination of the neural events to be detected and utilised. There are many well-known and easily-detectable neural events described in the literature, such as visual evoked potentials, slow cortical potentials, P300 events and imagined movement. Detecting these events relies on the known response of the brain to either external sensory inputs or internal thought processes.

OzCHI 2007, 28-30 November 2007, Adelaide, Australia. Copyright the author(s) and CHISIG. Additional copies are available at the ACM Digital Library (http://portal.acm.org/dl.cfm) or can be ordered from CHISIG(secretary@ chisig.org)

OzCHI 2007 Proceedings, ISBN 978-1-59593-872-5
In most cases, these neural events are relatively easy to detect by electroencephalogram (EEG) measurements, and robust classification algorithms exist for many of them (see, for example [7]). However, the neural events which form the basis of this type of human-computer communication often have very little to do with the goals or interaction metaphors of the interface itself. For example, in selecting letters for text composition, participants may be required to repeatedly imagine moving their left or right feet [5]. We denote these types of interfaces as "artificial" BCIs.

At the other end of the BCI spectrum are designs where there is a direct and obvious relationship between the neural events to be detected and the purpose of the interface. These "natural" BCIs could be techniques such as "imagining walking" to "really walk" through a virtual environment [8] or they could be more abstract measures of cognitive processes such as attention, emotion and creativity.

BCIs offer exciting possibilities for electronic music composition and performance. Measurements of neural activity can be used either to complement or to replace musical input devices such as MIDI controller keyboards. Musical BCI technology, while still in its infancy, has already been demonstrated to be a viable approach to music creation [9] although existing systems have primarily taken an artificial BCI approach: A participant, or "biomusician", is trained to perform standard artificial BCI "tricks", and the resulting control information is used to control or modulate the output of a music generation engine.

In this paper, we describe a novel approach to the design of a music BCI which differs from other musical BCIs in the types of neural activity which are detected and how they are used to generate music. This new approach is based on measurements of functional connectivity between brain regions which is discussed in the next section. This section also describes a new real-time technique to derive a measure of functional connectivity. The Mind Attention Interface, which is the target implementation environment for our system is described in Section 3. Finally, the computer music instrument is described in Section 4.

\section{FUNCTIONAL CONNECTIVITY AND MUSIC PROCESSING IN THE BRAIN}

Functional connectivity describes the temporary and taskdependent connections between different brain regions [14]. These connections are primarily governed by the integration of distributed local-processing assemblies in the brain to form higher-level processing networks. Functional con- 
nectivity in the brain is characterised by synchronous activation patterns across different brain regions. There are several methods for measuring functional connectivity and a quantitative comparison can be found in [3].

Evidence of functional connectivity as an indication of musical processing activity in the brain has been discovered in [4]. In this study, musicians and non-musicians were monitored using EEG while listening to music. The EEG signals were narrow-band filtered into frequency bands known to be of interest and the functional connectivity in each frequency band was measured. The results showed a statistically significant difference between the two groups in the gamma frequency band $(30-50 \mathrm{~Hz})$ and it was suggested that this could be due to the musician's increased musical processing ability relative to the non-musicians. The dramatic nature of the evidence presented in this study gives rise to the notion of using real-time measures of functional connectivity as a measure of attention in the feedback loop of the Mind Attention Interface and recent developments in digital signal processing appear to make such a system feasible as described below.

Given a discrete-time signal window $x[n]$ (of length $N$ ) which represents the neural activity measured at a given electrode site, it is useful to construct the associated "analytic signal" $z[n]=x[n]+i \mathscr{H}\{x[n]\}$ where $\mathscr{H}\{x[n]\}$ denotes the Hilbert Transform of $x[n]$. The analytic signal has the property that all the negative frequency components $(-\pi \leq \omega<0)$ of the signal have been filtered out. This can be computed efficiently in the frequency domain [11] using the Discrete Fourier Transform, $X[m]$, of the signal segment:

$$
Z[m]= \begin{cases}X[0], & \text { for } m=0 \\ 2 X[m], & \text { for } 1 \leq m \leq \frac{N}{2}-1 \\ X\left[\frac{N}{2}\right], & \text { for } m=\frac{N}{2} \\ 0, & \text { for } \frac{N}{2}+1 \leq m \leq N-1\end{cases}
$$

then the $N$-point inverse DFT is computed:

$$
z[n]=\frac{1}{N T} \sum_{m=0}^{N-1} Z[m] e^{i 2 \pi m n / N}
$$

to obtain the discrete-time analytic signal.

The brain can be modelled as a network of coupled chaotic oscillators. In this case, two oscillators are said to be synchronised when the weak locking condition

$$
\left|\phi_{1}-\phi_{2}\right| \bmod 2 \pi<\text { constant }
$$

is satisfied [12]. The instantaneous phase $\phi$ of a given (discretetime) signal $x[n]$ can be determined using the analytic signal. Considering the real and imaginary parts, and using the standard polar representation, the phase of the signal is then given by $\phi[n]=\tan ^{-1}(\mathscr{H}\{x[n]\} / x[n])$. This allows the calculation of the relative phase distribution $\phi_{i j}[n]$ between any pair of electrodes $i$ and $j$. This distribution is then compared to the $\delta$-distribution (which represents perfect synchrony). If we define

$$
\rho_{i j}=\frac{E_{\max }-E_{i j}}{E_{\max }}
$$

where $E$ is the Shannon's entropy of the distribution and $E_{\max }=0.626+0.4 \ln (N-1)$ (see [10] for details) then $\rho_{i j}$ is close to 0 when there is a low level of synchrony, and approaches 1 in the case of perfectly synchrony. Averaging this measure over all possible electrode pairs, we have a global measure of neural synchrony which can be evaluated over $K$ electrodes and a given signal window:

$$
\rho=\frac{2}{K(K-1)} \sum_{i=1}^{K} \sum_{j=i+1}^{K} \rho_{i j}
$$

Thus, $\rho$ provides a measure of the amount of synchronisation for that particular time window and, in this way, an overall index of functional connectivity can be obtained.

\section{THE MIND ATTENTION INTERFACE}

The "Mind Attention Interface" (MAI) is a flexible platform which is intended to support research into interactions between the human mind, human attention and virtual environments. It has been sited in a two-walled, walk-in virtual reality theatre similar to the CAVE which supports a spatialised 8.1 channel sound cube as well as computer graphics in mono or stereo. Inputs to the MAI system include a 16channel, Biosemi Active Two [1], active-electrode electroencephalogram (EEG) system and a Seeing Machines faceLab4 stereo camera system [2] (to track head and eye movements). One way of looking at the MAI is to say that the virtual reality theatre provides a feedback loop through the mind of the participant. The mind-modulated music system described in this paper is one of a number of "application layer" projects being developed for the MAI and it makes use of the EEG input and spatialised audio output capabilities of the installation as shown in Fig. 1.

The MAI application layer projects interface with a custombuilt software system (the "MAI System") which is made up of four system layers (device drivers, server, digital signal processing and clients). This MAI System presently coordinates the activities of some 5 computers over a local area network and it is compatible with other widely-used, open-source BCI systems. The signal acquisition approach has been to construct a signal processing engine (the "MAI Spectral Engine") in Matlab and Simulink, to run this engine on a dedicated computer using Matlab's xPC Target toolbox, and to connect this computer to our "MAI Server" (which coordinates the overall MAI System) using a customised connection kit. As described in the next section, our music generation engine has been implemented in impromptu [13], a dynamic live programming environment for Mac OS X which is described in the next section. Apple's Logic Pro software environment is also used for musical synthesis and sound spatialisation.

\section{A BIOMUSICIAN INTERFACE}

The biomusician interface which we have constructed can be thought of as one step towards the ultimate goal of harnessing natural musical processes in the brain to build a computer music instrument. The musical output of the system is not derived directly from the neural activity of the biomusician. The idea is that the musical attention (functional connectivity) in the biomusician's brain, as measured by $\rho$ in Section 2, can be used to inform the quality or characteristics of the generated music. To this end, it is necessary to have a music generation engine which can generate music which varies along some characteristic dimension. If we index this musical variation by some parameter $\chi$, then a mapping between $\chi$ and $\rho$ can be used to govern the evolution of the generated music. 


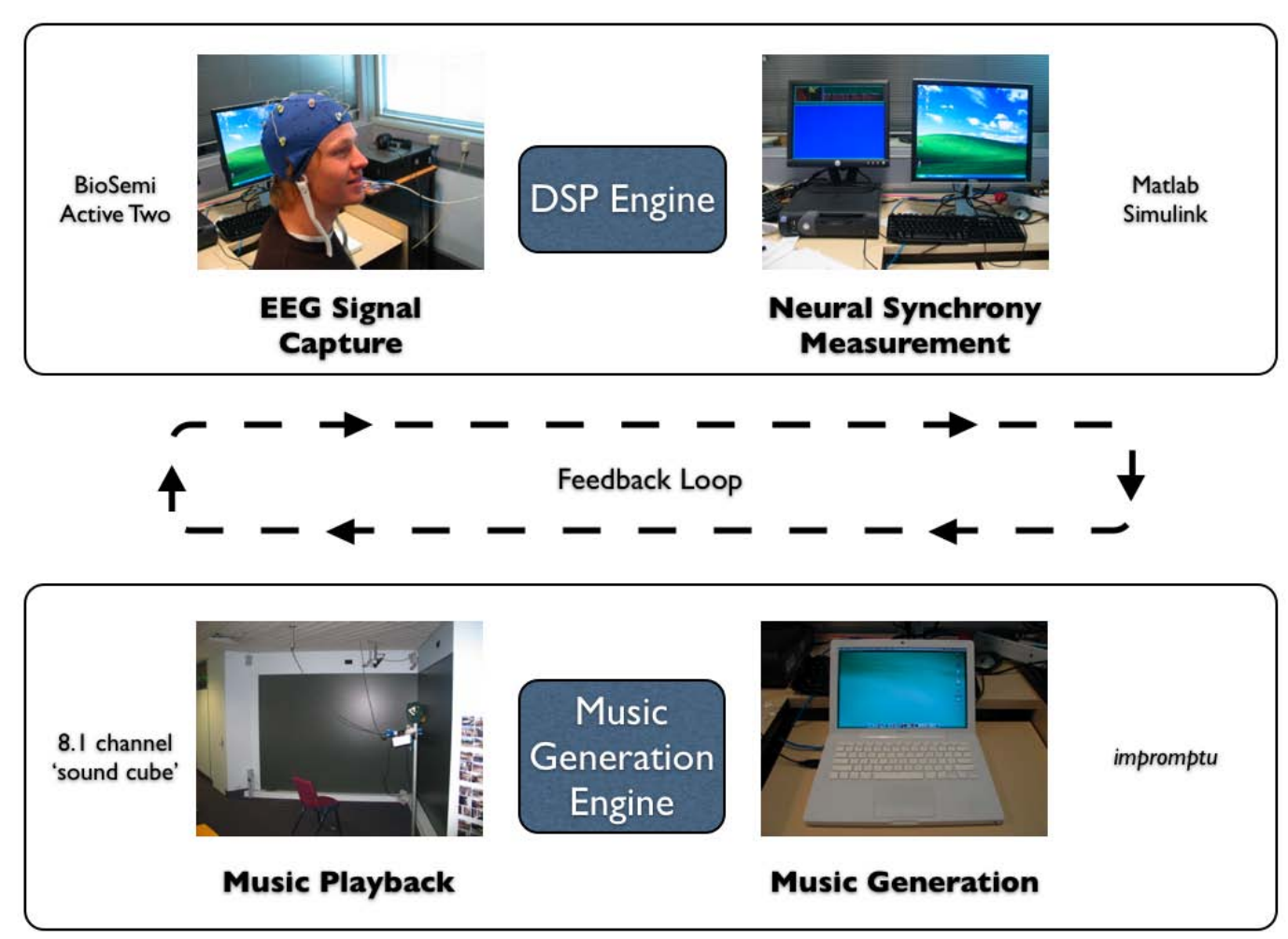

Figure 1: Feedback loop for mind-modulated music in the Mind Attention Interfce

One technique for indexing musical variation involves Euler's "Tonnetz" [6]. The Tonnetz is a topological representation of the 12 different notes of the musical scale. The Tonnetz is a pitch class $(C=0, C \sharp=1, \ldots)$ lattice, with travel in the horizontal and vertical directions occurring in \pm 4 and \pm 3 semitone steps, respectively. Pitch classes do not distinguish octaves, so the Tonnetz pitch class patterns repeat modulo 12 , and the structure stretches infinitely in all directions. A section of the Tonnetz is shown in Fig. 2. Its structure provides a quantifiable relationship between different harmonic triads, which are formed as shown in triangles formed by pitch-class triples.

In our system, the music engine generates music by taking biased random walks around the chord triads of the Tonnetz to generate chord progressions for improvisation. For a given root of the chord progression (shown as $\mathrm{C}$ and its relative minor, Am, in grey circles in Fig. 2), an appropriate scale for music generation is given by the black pitch classes. Furthermore, this scale is appropriate as long as the chord walk remains in the marked progression region. A parameter $\chi$ (which is driven by the functional connectivity measure $\rho$ ) governs the likelihood of the harmonic walk to stay within this region. When $\chi$ is small, the chords chosen remain close to the tonic. As $\chi$ increases, the harmonic walk becomes more expansive. Once the walk strays outside the region, the current chord becomes the new root of the progression, and the scale for improvisation is redefined.

Using this method, a biomusician can control a musical stimulus merely by attending to it (or concentrating on it). In this way, the natural musical sensibilities and cognitive tools of the musician are responsible for the modulation of the music.

The music generated engine in our system has been implemented in impromptu. Impromptu, written by Andrew Sorensen, is a real-time MIDI manipulation environment with sample-accurate temporal scheduling. It utilises a Scheme interpreter for algorithmic development, and Apple Computer's CoreAudio framework for MIDI routing. Live performances using impromptu often feature a composer who programs "dynamically" while the music is looping. The approach is compatible with semi-repetitive music, such as minimalist or dance music, which responds to modulations in one of its dimensions.

The present status of our project is that the biomusician interface has been completed and it is currently undergoing testing within the Mind Attention Interface. In the near future, we plan to test the system using volunteers with substantial musical ability. Apart from discovering general usability issues in the interface, the objective of these tests will be to ascertain whether the test participants can distinguish between the operation of the functional-connectivity based musical generator and a "placebo" which is based on qualitatively similar, but random, behaviour.

\section{ACKNOWLEDGEMENTS}

Ben Swift and James Sheridan gratefully acknowledge the support of the Apple University Consortium (AUC).

\section{REFERENCES}




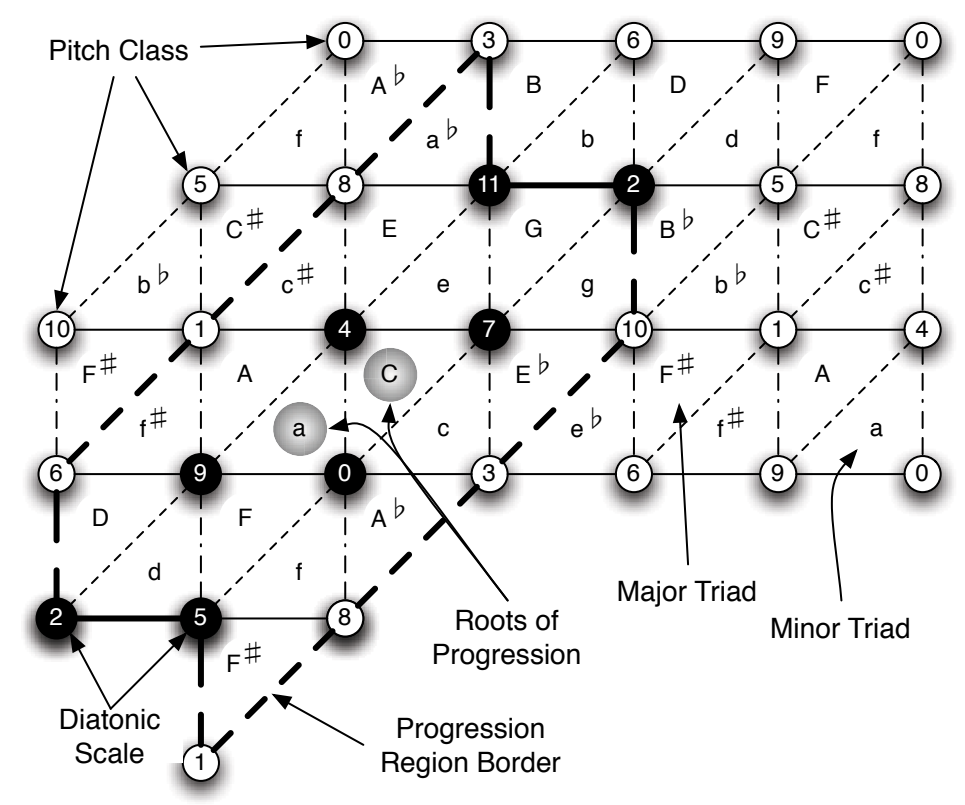

Figure 2: A representation of the Tonnetz

[1] Biosemi Active Two. www.biosemi.com/products.htm, accessed September 2007.

[2] SeeingMachines faceLAB 4. www.seeingmachines.com/facelab.htm, accessed September 2007.

[3] K. Ansari-Asl, L. Senhadji, J. Bellanger, and F. Wendling. Quantitative evaluation of linear and nonlinear methods characterizing interdependencies between brain signals. Physical Review E, 74(3):31916, 2006.

[4] J. Bhattacharya and H. Petsche. Phase synchrony analysis of EEG during music perception reveals changes in functional connectivity due to musical expertise. Signal Processing, 85(11):2161-2177, 2005.

[5] B. Blankertz, G. Dornhege, M. Krauledat, M. Schroeder, J. Williamson, R. Murray-Smith, and K. Müller. The Berlin Brain-Computer Interface presents the novel mental typewriter Hex-o-Spell.

[6] E. Chew. A walk through tonal space: Charting the establishing of key of a melody. Proceedings of the 3rd Annual Symposium on Systems Research in the Arts, Baden-Baden, Germany, August, 2001.

[7] X. Gao, D. Xu, M. Cheng, and S. Gao. A BCI-based environmental controller for the motion-disabled. Neural Systems and Rehabilitation Engineering, IEEE Transactions on [see also IEEE Trans. on Rehabilitation Engineering], 11(2):137-140, 2003.

[8] R. Leeb, C. Keinrath, D. Friedman, C. Guger, R. Scherer, C. Neuper, M. Garau, A. Antley, A. Steed, M. Slater, et al. Walking by thinking: the brainwaves are crucial, not the muscles! Presence: Teleoperators and Virtual Environments, 15(5):500-514, 2006.

[9] E. Miranda and A. Brouse. Toward direct brain-computer musical interfaces. Proceedings of the 2005 conference on New interfaces for musical expression, pages 216-219, 2005.

[10] R. Otnes and L. Enochson. Digital Time Series Analysis. John Wiley \& Sons, Inc. New York, NY, USA, 1972.

[11] A. Reilly and G. Frazer. An efficient algorithm for analytic signal generation fortime-frequency distributions. Acoustics, Speech, and Signal Processing, 1994. ICASSP-94., 1994 IEEE International Conference on, 3, 1994.

[12] M. Rosenblum, A. Pikovsky, and J. Kurths. Phase Synchronization of Chaotic Oscillators. Physical Review Letters, 76(11):1804-1807, 1996.

[13] A. Sorensen. Impromptu: An interactive programming environment for composition and performance. Proceedings of the Australasian Computer Music Conference 2005, pages 149-153.

[14] C. Stam and E. de Bruin. Scale-free dynamics of global functional connectivity in the human brain. Human Brain Mapping, 22(2):97-109, 2004.

[15] J. Wolpaw, N. Birbaumer, D. McFarland, G. Pfurtscheller, and T. Vaughan. Brain-computer interfaces for communication and control. Clinical Neurophysiology, 113(6):767-791, 2002. 